\title{
Growth Equations: \\ A General Equation and a Survey of Special Cases
}

\author{
MICHAEL A. SAVAGEAU \\ Department of Microbiology and Immunology, \\ The University of Michigan, Ann Arbor, Michigan 48109
}

Received 2 September 1979

\begin{abstract}
Although growth in its various manifestations has been studied for centuries and although there are a large number of well-established growth "laws," that work is almost entirely empirical and lacks a theoretical foundation with which macroscopic aspects of growth might be related to underlying, microscopic determinants. Recent work on the analysis of complex systems, however, has provided just such a foundation. It has been shown that an important class of complex systems can be accurately described by a formalism involving simple nonlinear approximations. This formalism leads naturally to a general growth equation in differential form for complex systems. The survey of wellestablished growth equations presented here demonstrates that each of these is a special case of the general growth equation.
\end{abstract}

\section{INTRODUCTION}

The mathematical characterization of growth is among the oldest scientific pursuits [35] and transcends most modern, often narrowly defined, disciplines. The literature abounds with specific growth equations of demonstrated utility. Yet these equations are all empirically derived and lack a fundamental, theoretical relationship to the underlying mechanisms of growth. Recently I have derived a general growth equation that is based upon the nature of the elemental mechanisms in complex systems [35]. Here I shall briefly describe this general equation and then show that it has as special cases all the well-established growth equations.

\section{GENERAL GROWTH EQUATION}

Theoretically, the behavior of a complex system can be related to the nature of its component mechanisms. The prerequisite for obtaining this relation is a knowledge of the elementary descriptions for each of the component mechanisms. The mathematical form of these descriptions has 
been presented elsewhere for a broad class of mechanisms called synergistic [33-35]. These component descriptions can be combined to yield a set of differential equations capable of representing the intact system.

For a system composed of $n$ elements, different in kind and/or location, the symbol $X_{i}$ has been used to represent the concentration or amount of the $i$ th element. $\dot{X}_{i}$ denotes the time derivative of $X_{i}$. By suitable numbering from 1 to $n$, the subscripts ( $i$ ) can be used to denote both the identity and the location of an element. Additional variables with $i$ varying from $n+1$ to $s$ can be defined as aggregate measures of the entire system and of particular subsystems within the system. For example, these could be the total weight of an organism, or a particular organ of the organism; the total population of a society, or a particular group within the society; the capital accumulation of an entire economy, or of a particular sector of that economy, etc. Each aggregate measure is the sum of all the relevant elements of the system or subsystem, i.e.,

$$
X_{i}=\sum_{\substack{\text { relevant } \\ j \text { from } 1}}^{n} X_{j}, \quad i=n+1, n+2, \ldots, s .
$$

With the conventions in the preceding paragraph, it can be shown [34, 35 ] that the behavior of complex synergistic systems is determined by the nature of the component mechanisms and their abundant interrelationships, as expressed mathematically in the following equations:

$$
\dot{X}_{i}=\alpha_{i} \prod_{j=1}^{n} X_{j}^{g_{i j}}-\beta_{i} \prod_{j=1}^{n} X_{j}^{h_{i j}}, \quad i=1,2, \ldots, s
$$

and

$$
X_{i}=\gamma_{i} \prod_{j=1}^{n} X_{j}^{f_{i j}}, \quad i=n+1, n+2, \ldots, s
$$

The problem of analyzing such systems can be viewed as a process of extracting the information latent in these descriptive equations.

By starting with Eqs. (2) and (3) and a single reasonable postulate, one can derive a general growth equation [35]. The postulate is that most changes among the component parts of a system occur much faster than the rate of growth for the system as a whole. Mathematically, this implies that a 
small number $(k)$ of the equations (2), representing the slowest phenomena, determine the temporal response of the entire system; all other equations, representing the faster phenomena, can be assumed to have reached a quasisteady state with time derivatives equal to zero. Under these conditions, the descriptive equations can be reduced to the following $k$ equations:

$$
\dot{X}_{i}=\alpha_{i} \prod_{j=1}^{k} X_{j}^{g_{i j}}-\beta_{i} \prod_{j=1}^{k} X_{j}^{h_{i j}}, \quad i=1,2, \ldots, k
$$

in which an $X_{i}$ may represent either one of the $n$ fundamental variables or one of the $s-n$ aggregate measures, including the total system.

Thus, Eq. (4) represents a general growth equation in differential form for the entire system when there are $k$ temporally dominant processes. Most systems appear to be governed by a very small number of temporally dominant equations. In fact, as will be seen in the next two sections, all the well-known growth equations are special cases of the equations (4) when there are only one or two temporally dominant equations. ${ }^{1}$

\section{GROWTH EQUATIONS IN ONE VARIABLE}

When there is a single, temporally dominant process, the basic growth equation (4) can be written

$$
\dot{X}_{1}=\alpha_{1} X_{1}^{\beta_{11}}-\beta_{1} X_{1}^{h_{11}}
$$

There are several well-known growth equations that are special cases of Eq. (5). A summary of the following comparisons is presented in Table 1.

\footnotetext{
'There is additional evidence, which I will present elsewhere [36, 37], that supports the notion of a small number of temporally dominant processes in complex systems. Any system that grows into a stable mature form has a growth curve that is a legitimate cumulative probability distribution. Conversely, the integral function of any probability distribution exhibits properties of limited growth. Thus, there are an enormous number of cumulative probability distributions that also represent potential growth functions. I have found that nearly all the well-known cumulative probability distributions can be represented by the general growth equation (4) when there are a small number of temporally dominant processes [37]. Furthermore, the well-known allometric growth relationships can be derived from Eqs. (2) and (3) when there are a small number of temporally dominant equations [36].
} 
TABLE 1

Comparison of the Basic Growth Equation in a Single Variable and Several Special Cases

\begin{tabular}{|c|c|c|c|c|c|}
\hline \multirow{2}{*}{$\frac{\text { Growth law }}{\text { Basic }^{\mathrm{a}}}$} & \multicolumn{4}{|c|}{ Parameters } & \multirow{2}{*}{ References } \\
\hline & $\alpha_{1}$ & $g_{11}$ & $\beta_{1}$ & $h_{11}$ & \\
\hline Linear & $C$ & 0 & 0 & 0 & 32,41 \\
\hline Exponential & $k$ & 1 & 0 & 0 & $4,18,32$ \\
\hline Monomolecular & $k w_{f}$ & 0 & $k$ & 1 & $1,2,32$ \\
\hline Logistic & $r$ & 1 & $r / K$ & 2 & $\begin{array}{l}1,3,12,21,26, \\
31,32,39,44\end{array}$ \\
\hline Bertalanffy & $\eta$ & $m$ & $\kappa$ & 1 & 1 \\
\hline
\end{tabular}

${ }^{\mathrm{a}} \dot{X}_{1}=\alpha_{1} X_{1}^{g_{11}}-\beta_{1} X_{1}^{h_{11}}$.

\section{LINEAR}

Aside from a small number of examples in which growth is naturally linear [32], linear growth may result whenever an essential factor for growth is limiting and conserved at a constant value. For example, when a gene essential for the growth of a microorganism is carried on an extrachromosomal element that cannot be replicated nor integrated into another replicating element, the growth of the population becomes linear. The cells having the essential gene grow relatively normally, but at the time of cell division only one of their two daughter cells can receive the essential gene; the other becomes "sterile."2 The equation for linear growth is

$$
N(t)=C(t+a)
$$

and in differential form

$$
\frac{d N}{d t}=C
$$

where $N(t)$ is number at time $t, C$ is the growth-rate constant, and $a=N(0) / C$ is related to the number at $t=0$. In terms of Eq. (5): $\alpha_{1}=C$, and all other parameters have the value zero.

\section{EXPONENTIAL}

The law of exponential growth, or the law of Malthus [20] as it is sometimes called, is probably the best-known form of growth. Within limits, it applies extremely well to a wide variety of systems, e.g., microorganisms

\footnotetext{
${ }^{2}$ For a further discussion of this and other examples, see Ref. [41].
} 
[4], plants [32], tumors (see Ref. [18]), publications on Drosophila [9], and manufacturing companies [8]. In conventional notation,

$$
N(t)=N(0) \exp (k t)
$$

and in differential form

$$
\frac{d N}{d t}=k N
$$

where $N(t)$ is number at time $t$, and $k$ is the exponential growth-rate constant. In the notation of Eq. (5): $\alpha_{1}=k, g_{11}=1$, and all other parameters have the value zero.

\section{MONOMOLECULAR (LIMITED OR DECREASING EXPONENTIAL)}

This equation describes the growth of certain animals during most of their lives $[1,2]$ and certain plants [32]; it can be written in the form

$$
w=w_{f}[1-b \exp (-k t)]
$$

where $w$ is weight or volume as a function of time, $w_{f}$ is the final value of $w$, $k$ is the growth-rate constant, and $b$ is a positive parameter related to the initial value of $w$.

In differential form,

$$
\frac{d w}{d t}=k\left(w_{f}-w\right)
$$

and the parameters of the basic growth equation (5) can be identified as follows: $\alpha_{1}=k w_{f}, \beta_{1}=k, g_{11}=0$, and $h_{11}=1$.

\section{LOGISTIC}

The logistic equation was proposed by the mathematician Verhulst [42] and since has become one of the most popular equations for describing growth of animals [1, 21], plants [32], populations [26, 31, 39], and economies [3, 12]. (For a criticism of some of the applications of the logistic and related functions, see Ref. [1].) The equation is often written

$$
N(t)=K\left[1+\exp \left(\frac{r}{K}-r t\right)\right]^{-1}
$$

where $N(t)$ is number or amount as a function of time, $K$ is the final value of $N$, and $r$ is the growth-rate constant. In differential form the logistic equation becomes

$$
\frac{d N}{d t}=r N-\frac{r}{K} N^{2}
$$


and thus the parameters in the basic growth equation (5) are $\alpha_{1}=r$, $\beta_{1}=r / K, g_{11}=1$, and $h_{11}=2$.

\section{BERTALANFFY}

This is one of the most successful equations for describing animal growth because there has been an attempt to relate the overall growth to the physiology of the organism and because of the diversity of growth data that are well described. The advantages and limitations of this equation have been discussed extensively by Bertalanffy [1]. Although the equation can be written as

$$
w(t)=\left[\frac{\eta}{\kappa}-\left(\frac{\eta}{\kappa}-w_{0}^{1-m}\right) e^{-(1-m) \kappa t}\right]^{1 /(1-m)},
$$

the differential form is more common and more easily understood:

$$
\frac{d w}{d t}=\eta w^{m}-\kappa w
$$

where $w$ is weight or amount as a function of time; $\kappa$ is a first-order rate constant for loss due to catabolism, which is assumed to be proportional to $w$ or body mass; and $\eta$ and $m$ are parameters of a power law describing anabolism, which is assumed to be proportional to the surface area (hence, the power function of mass or volume) through which nutrients are absorbed [1]. In the notation of the basic equation (5): $\alpha_{1}=\eta, \beta_{1}=\kappa, g_{11}=m$, and $h_{11}=1$.

\section{GROWTH EQUATIONS IN TWO VARIABLES}

When there are two temporally dominant processes, the basic growth equation (4) can be written

$$
\begin{aligned}
& \dot{X}_{1}=\alpha_{1} X_{1}^{g_{11}} X_{2}^{g_{12}}-\beta_{1} X_{1}^{h_{11}} X_{2}^{h_{12}}, \\
& \dot{X}_{2}=\alpha_{2} X_{1}^{g_{21}} X_{2}^{g_{22}}-\beta_{2} X_{1}^{h_{21}} X_{2}^{h_{22}}
\end{aligned}
$$

The remaining growth equations considered in this survey are special cases of Eq. (6). The results of the following comparisons are summarized in Table 2. 
TABLE 2

Comparison of the Basic Growth Equation in Two Variables and Several Special Cases

\begin{tabular}{lcccccccccccccc}
\hline Growth Law & \multicolumn{10}{c}{ Parameters } \\
\hline Basic & $\alpha_{1}$ & $g_{11}$ & $g_{12}$ & $\beta_{1}$ & $h_{11}$ & $h_{12}$ & $\alpha_{2}$ & $g_{21}$ & $g_{22}$ & $\beta_{2}$ & $h_{21}$ & $h_{22}$ & - \\
Logarithmic & $b$ & 0 & 1 & 0 & 0 & 0 & 0 & 0 & 0 & 1 & 0 & 2 & 6 \\
Power law & $b c$ & 0 & $1-b$ & 0 & 0 & 0 & 0 & 0 & 0 & 1 & 0 & 2 & $1,5,25$, \\
& & & & & & & & & & & & & 32,40 \\
Hyperbolic & $b$ & 0 & 2 & 0 & 0 & 0 & 0 & 0 & 0 & $a^{-1 / 2}$ & 0 & 2 & 22 \\
Weibull & $c k$ & 1 & $c+1$ & 0 & 0 & 0 & 0 & 0 & 0 & 1 & 0 & 2 & 46,47 \\
Stochastic & $2 k_{g}$ & 1 & $\frac{1}{2}$ & 0 & 0 & 0 & 0 & 0 & 0 & 1 & 0 & $\frac{1}{2}$ & 7,11 \\
Gompertz & 1 & 1 & 1 & 0 & 0 & 0 & 0 & 0 & 0 & $\alpha$ & 0 & 1 & $1,10,17$, \\
Lotka-Volterra & $r$ & 1 & 0 & $k$ & 1 & 1 & $K$ & 1 & 1 & $d$ & 0 & 1 & 19,45 \\
\hline$\dot{X}_{1}=\alpha_{1} X_{1}^{g_{11}} X_{2}^{g_{12}}-\beta_{1} X_{1}^{h_{11}} X_{2}^{h_{12}} ; \dot{X}_{2}=\alpha_{2} X_{1}^{g_{21}} X_{2}^{g_{22}}-\beta_{2} X_{1}^{h_{21}} X_{2}^{h_{22}}$. & & & &
\end{tabular}

\section{LOGARITHMIC}

This type of equation is sometimes used to describe the decelerating phase of animal growth in certain species [6]. The equation can be written

$$
w(t)=c+b \log (t+a)
$$

or

$$
\frac{d w}{d t}=\frac{b}{t+a},
$$

where $w$ is weight or amount as a function of time, and $a, b$, and $c$ are positive constants. This equation is equivalent to the following set of equations in which time no longer appears as an explicit variable ${ }^{3}$ :

$$
\begin{aligned}
& \dot{w}=b z, \\
& \dot{z}=-z^{2} .
\end{aligned}
$$

From these equations the parameters in the basic equation (6) can be identified: $\alpha_{1}=b, \beta_{2}=1, g_{12}=1, h_{22}=2$, and all others have the value zero.

${ }^{3}$ The differential form of this growth equation, and most of those that follow, is not unique. For example, an alternative description of the logarithmic growth equation is

$$
\begin{aligned}
& \dot{w}=b z^{n} \\
& \dot{z}=-z^{n+1} / n, \quad n>0 .
\end{aligned}
$$


POWER LAW

Special cases of this equation have been proposed for the growth of various organisms $[1,32]$ and tumors $[5,24,25]$. The equation can be written

$$
w(t)=c(t+a)^{b}
$$

and in differential form

$$
\begin{aligned}
& \dot{w}=b c z^{1-b}, \\
& \dot{z}=-z^{2},
\end{aligned}
$$

where again $w$ is weight or amount as a function of time, and $a, b$, and $c$ are positive constants in the equation. Comparing these equations with Eq. (6) shows that $\alpha_{1}=b c, \beta_{2}=1, g_{12}=1-b$, and $h_{22}=2$; the other parameters in Eq. (6) have the value zero.

\section{HYPERBOLIC}

Regenerative growth in some instances has been represented by a hyperbolic function [40]. A generalized form of this function that is capable of representing certain S-shaped or sigmoid growth curves can be written

$$
w(t)=\frac{w_{f}(t+a)^{n}}{b+(t+a)^{n}}
$$

where $w$ is weight or amount as a function of time, $w_{f}$ is the final value of $w$, and $a, b$, and $n$ are positive parameters in the equation. In differential form

$$
\begin{aligned}
\dot{w} & =b n w^{2} z^{n+1}, \\
\dot{z} & =-z^{2},
\end{aligned}
$$

and the corresponding values of the parameters in the basic growth equation (6) are: $\alpha_{1}=b n, \beta_{2}=1, g_{11}=2, g_{12}=n+1, h_{22}=2$, and all other parameters have the value zero.

This degeneracy is to be expected because $z$ represents only one of a finite number of choices for any given system and because there are an infinite number of systems that can yield the same growth equation. Nevertheless, there are certain restrictions that must be obeyed. Since $z$ represents the amount or concentration of an element of the system, its value cannot become negative or infinite; this restricts the possibilities for the second differential equation. This equation can be specified more precisely with additional detailed information about the mechanisms underlying growth. 
WEIBULL

Another growth equation that has been suggested to have widespread applicability [22] was originally developed by Weibull [46]. It can be written

$$
w(t)=w(0) \exp \left[-k(t+a)^{-c}\right],
$$

where $w$ is weight or amount as a function of time, and $a, c$, and $k$ are positive parameters. In differential form, this equation can be written

$$
\begin{aligned}
\dot{w} & =c k w z^{c+1}, \\
\dot{z} & =-z^{2},
\end{aligned}
$$

and the parameters that have values different from zero in the basic growth equation (6) can be identified as: $\alpha_{1}=c k, \beta_{2}=1, g_{11}=1, g_{12}=(c+1)$, and $h_{22}=2$.

\section{STOCHASTIC}

Zotina and Zotin [47] have developed a growth equation based on the normal probability distribution - hence the name stochastic. In its general form, this growth equation also includes powers of the normal distribution. The growth of certain insects is well described by this equation (see Refs. $[11,47])$. In the notation of Zotina and Zotin [47]

$$
w(t)=w_{m} \exp \left(-\frac{k_{\mathrm{g}}}{2}\left(T_{m}-t\right)^{2}\right),
$$

where $w$ is weight or amount as a function of time, $w_{m}$ is the maximum value of $w, T_{m}$ is the time when growth stops, and $k_{g}$ is the growth-rate constant. In differential form this equation can be written

$$
\begin{aligned}
\dot{w} & =2 k_{g} w z^{1 / 2}, \\
\dot{z} & =-z^{1 / 2},
\end{aligned}
$$

and the parameters of the basic equation (6) are seen to be: $\alpha_{1}=2 k_{8}, \beta_{2}=1$, $g_{11}=1, g_{12}=\frac{1}{2}, h_{22}=\frac{1}{2}$, and all other parameters have the value zero.

\section{GOMPERTZ}

This equation, proposed by Gompertz [7] for actuarial tables, has become one of the most popular for describing growth of animals $[1,15,17]$, embryos [14], plants [32], tumors [10,13, 16, 23], and populations of organisms [31,39], and is still used in studies of aging and mortality [31, 39]. In the notation of Laird [16]

$$
V(t)=V(0) \exp [-\beta \exp (-\alpha t)]
$$


where $V$ is volume or weight as a function of time, and $\alpha$ and $\beta$ are positive parameters. In differential form

$$
\begin{aligned}
& \dot{V}=k V, \\
& \dot{k}=-\alpha k .
\end{aligned}
$$

Thus, the parameters of the basic equation (6) that have values different from zero are: $\alpha_{1}=1, \beta_{2}=\alpha, g_{11}=1, g_{12}=1$, and $h_{22}=1$.

\section{LOTKA-VOLTERRA}

The following equations are the classic ones studied by Lotka [19] and Volterra [45]; they represent the growth in differential form of two populations in a predator-prey relationship:

$$
\begin{aligned}
& \dot{X}=r X-k X Y, \\
& \dot{Y}=K X Y-d Y,
\end{aligned}
$$

where $X$ and $Y$ represent the sizes of the prey and predator populations, respectively, and all the parameters are positive in value. The corresponding values for the parameters of the basic equation (6) are: $\alpha_{1}=r, \alpha_{2}=K$, $\beta_{1}=k, \beta_{2}=d, g_{11}=1, g_{12}=0, g_{21}=1, g_{22}=1, h_{11}=1, h_{12}=1, h_{21}=0$, and $h_{22}=1$.

\section{DISCUSSION}

There are still more growth equations (e.g., those discussed by Turner et al. $[42,43]$ and by Peil and his colleagues $[27-30,38]$ in a series of papers on growth) that could be considered here, but suffice it to say these are special cases of the basic growth equation (4) when there are two or more temporally dominant processes. Since there are numerous examples of data that are well characterized by each of the specialized laws of growth, there clearly are an enormous number of examples that are described by the basic growth equation (4). Although many, if not all, processes exhibiting "regular" growth are represented by these specialized growth equations, which as we have just seen are special cases of the basic growth equation (4), there are other processes that exhibit irregular growth curves with sharp breaks, oscillations, etc. The basic growth equation (4) has the potential for describing these processes as well.

This work was done in 1976-77 while I was the recipient of a fellowship from the John Simon Guggenheim Memorial Foundation. It also was supported in part by a grant from the National Science Foundation (BMS 75-01591). I thank Robert Rosen and A. M. Kotre for helpful criticism of the original manuscript. 


\section{REFERENCES}

1 L. von Bertalanffy, Principles and theory of growth, in Fundamental Aspects of Normal and Malignant Growth (W. W. Nowinski Ed.), Elsevier, New York, 1960, pp. 137-259.

2 S. Brody, Bioenergetics and Growth, Hafner, New York, 1964.

3 H. T. Davis, The Analysis of Economic Time Series, Reissued ed., Principia Press of Trinity Univ., San Antonio, Texas, 1963.

4 P. S. S. Dawson (Ed.), Microbial Growth, Dowden, Hutchinson and Ross, Stroudsburg, Penn., 1974.

5 L. A. Dethlefsen, J. M. S. Prewitt, and M. L. Mendelsohn, Analysis of tumor growth curves, J. Nat. Cancer Inst. 40:389-405 (1968).

6 J. G. Fleagle and K. W. Samonds, Physical growth of Cebus monkeys (Cebus albifrons) during the first year of life, Growth 39:35-52 (1975).

7 B. Gompertz, On the nature of the function expressive of the law of human mortality, Philos. Trans. Roy. Soc. London 36:513-585 (1825).

8 M. Haire, Biological models and empirical histories of the growth of organizations, in Modern Organization Theory (M. Haire, Ed.), Wiley, New York, 1959, pp. 272-306.

9 A. H. Hersh, Drosophila and the course of research, Ohio J. Sci. 42:198-200 (1942).

10 W. R. Inch, J. A. McCredie, and R. M. Sutherland, Growth of nodular carcinomas in rodents compared with multi-cell spheroids in tissue culture, Growth 34:271-282 (1970).

11 A. W. Knight, M. A. Simmons, and C. S. Simmons, A phenomenological approach to the growth of the winter stonefly, Taeniopteryx nivalis (Fitch) (Plecoptera: Taeniopterygidac), Growth 40:343-367 (1976).

12 S. S. Kuznets, Secular Movements in Production and Prices, Augustus M. Kelley Publishers, New York, 1967.

13 A. K. Laird, Dynamics of tumor growth: comparison of growth rates and extrapolation of growth curve to one cell, British J. Cancer 19:278-291 (1965).

14 A. K. Laird, Dynamics of embryonic growth, Growth 30:263-275 (1966).

15 A. K. Laird, Postnatal growth of birds and mammals, Growth 30:349-363 (1966).

16 A. K. Laird, Dynamics of growth in tumors and in normal organisms, Nat. Cancer Inst. Monog. 30: 15-27 (1969).

17 A. K. Laird, S. A. Tyler, and A. D. Barton, Dynamics of normal growth, Growth 29:233-248 (1965).

18 C. Lightdale and M. Lipkin, Cell division and tumor growth, in Cancer, Vol. 3 (F. F. Becker, Ed.), Plenum, New York, 1975, pp. 201-215.

19 A. J. Lotka, Elements of Physical Biology, Williams \& Wilkins, Baltimore. 1924. (Reprinted as Elements of Mathematical Biology, Dover, New York, 1956.)

20 T. R. Malthus, An Essay on the Principle of Population, London, 1798. (Reprinted by St. Martin's Press, New York, 1966).

21 E. Marubini, L. F. Resele, J. M. Tanner, and R. H. Whitehouse, The fit of Gompertz and logistic curves to longitudinal data during adolescence on height, sitting height and biacromial diameter in boys and girls of the Harpenden growth study, Human Biol. 44:511-524 (1972).

22 N. G. McCormick, A proposal on nature's time-scale, Nature 208:334-336, (1965).

23 J. A. McCredie, W. R. Inch, J. Kruuv, and T. A. Watson, The rate of tumor growth in animals, Growth 29:331-347 (1965). 
24 M. L. Mendelsohn, Cell proliferation and tumor growth, in Cell Proliferation (L. F. Lamerton and R. J. M. Fry, Eds.), Davis, Philadelphia, 1963, pp. 190-210.

25 M. L. Mendelsohn and L. A. Dethlefsen, Tumor growth and cellular kinetics, in The Proliferation and Spread of Neoplastic Cells, Williams \& Wilkins, Baltimore, 1968, pp. 197-211.

26 R. Pearl, Studies in Human Biology, Williams \& Wilkins, Baltimore, 1924.

27 J. Peil, Mathematische Beschreibung von Wachstumsvorgängen, Gegenbaurs Morph. Jahrb., Leipzig 120:832-853 (1974).

28 J. Peil, Ansätze zur mathematischen Beschreibung von Wachstumsprozessen, Gegenbaurs Morph. Jahrb., Leipzig 121:163-173 (1975).

29 J. Peil and H. Helwin, Quantitative Beschreibung von Wachstumsvorgängen des Menschen als Grundlage objektiver Vergleiche von geschlechtsspezifischen Daten verschiedener Körpermasse, Gegenbuurs Morph. Jahrb., Leipzig 122:162-181 (1976).

30 J. Peil and H. Helwin, Quantitativ-analytische Erfassung des Körperlängenwachstums des Menschen, Gegenbaurs Morph. Jahrb., Leipzig 123:236-259 (1977).

31 R. Pressat, Demographic Analysis, Aldine-Atherton, Chicago, 1972.

32 F. J. Richards, The quantitative analysis of growth, in Plant Physiology, Vol. VA (F. C. Steward Ed.), Academic, New York, 1969, pp. 3-76.

33 M. A. Savageau, Biochemical systems analysis II. The steady state solution for an n-pool system using a power-law approximation, J. Theoret. Biol. 25:370-379 (1969).

34 M. A. Savageau, Biochemical Systems Analysis: A Study of Function and Design in Molecular Biology, Addison-Wesley, Reading, Mass. 1976.

35 M. A. Savageau, Growth of complex systems can be related to the properties of their underlying determinants, Proc. Nat. Acad. Sci. U.S.A. 76:5413-5417 (1979).

36 M. A. Savageau, Allometric morphogenesis of complex systems: a derivation of the basic equations from first principles, Proc. Nat. Acad. Sci. U.S.A. 76, (1979).

37 M. A. Savageau, A suprasystem of probability distributions, submitted for publication.

38 J. -H. Scharf and J. Peil, Ein Algorithmus zur Wertebestimmung der Parameter in der Gompertzschen Wachstumsfunktion, Gegenbaurs Morph. Jahrb., Leipzig 121:389-420 (1975).

39 H. S. Shryock, J. S. Siegel and Associates, The Methods and Materials of Demography, Vol 2, U.S. Government Printing Office, Washington, 1973.

40 R. P. Spencer and M. J. Coulombe, Quantitation of hepatic growth and regeneration, Growth 30:277-284 (1966).

41 R. Y. Stanier, E. A. Adelberg, and J. L. Ingraham, The Microbial World, 4th ed., Prentice-Hall, Englewood Cliffs, N.J., 1976, pp. 279, 491.

42 M. E. Turner, E. L. Bradley, K. A. Kirk, and K. M. Pruitt, A theory of growth, Math. Biosci. 29:367-373 (1976).

43 M. E. Turncr and K. M. Pruitt, A common basis for survival, growth and autocatalysis, Math Biosci. 39:113-123 (1978).

44 P. F. Verhulst, Notice sur la loi que la population suit dans son accroissement, Corr. Math. Phys. 10:113-121 (1838).

45 V. Volterra, Leçons sur la Théorie Mathématique de la Lutte pour la Vie, GauthierVillars, Paris, 1931.

46 W. Weibull, A statistical distribution function of wide applicability, J. Appl. Mech. 18:293-297 (1951).

47 R. S. Zotina and A. I. Zotin, Towards a phenomenological theory of growth, $J$. Theoret. Biol. 35:213-225 (1972). 